\title{
GEOMETRIC STRUCTURE OF SINGLE/COMBINED EQUIVALENCE CLASSES OF A CONTROLLABLE PAIR*
}

\author{
ALBERT COMPTA $^{\dagger}$, JOSEP FERRER $^{\dagger}$, AND MARTA PEÑA $^{\dagger}$
}

\begin{abstract}
Given a pair of matrices representing a controllable linear system, its equivalence classes by the single or combined action of feedbacks, change of state and input variables, as well as their intersection are studied. In particular, it is proved that they are differentiable manifolds and their dimensions are computed. Some remarks concerning the effect of different kinds of feedbacks are derived.
\end{abstract}

Key words. Controllable pairs, Linear systems, Orbits by feedback, Orbits by variables change, System perturbations.

AMS subject classifications. 37A20, 93C05, 93C 73 .

1. Introduction. Several equivalence relations between pairs of matrices representing linear systems are considered in the literature. For example, the one corresponding to a change of basis in the state variables, or the so-called block similarity, which also involves changes in the input space and feedbacks. It seems natural to consider the equivalence classes related to each one of these transformations and to several of them. We study the geometric relations between the different equivalence classes, such as the relative codimension and especially their nontrivial intersections.

Partial approaches to this subject appear in the literature (see, for example, [1], [3], [7], [14], [15]). Here we tackle a unified treatment in order to simplify the proofs and to present a full panorama of the geometric hierarchy of these equivalence classes. Some surprising remarks concerning the effect of the feedbacks will be derived.

The starting point is the differentiable structure of each equivalence class, which follows from the Closed Orbit Lemma (see for example [10]). The computation of its dimension is based on Arnold's technique of the so-called versal deformations, that is to say, transversal manifolds to the considered classes (or orbits) in some other coarser one. In fact, we use the results in [3] and [15] to obtain "adapted" deformations having similar patterns, in such a way that different families of parameters are responsible for

${ }^{*}$ Received by the editors on October 2, 2010. Accepted for publication on October 24, 2011. Handling Editor: Harm Bart.

${ }^{\dagger}$ Departament de Matemàtica Aplicada I, UPC, Diagonal 647, 08028 Barcelona, Spain (albert.compta@upc.edu, josep.ferrer@upc.edu, marta.penya@upc.edu). Partially supported by DGICYT MTM2010-19356-C02-02. 
the corresponding deformation. Moreover, it gives a local adapted parameterization of the different equivalence classes.

Regarding orbit intersections, they may not be orbits, not even differentiable manifolds. In our case, these intersections are differentiable manifolds because the transversality conditions hold and it is possible to give a particular description as orbits with regard to suitable subgroups. Even more, in some cases this subgroup is just the intersection of those generating the intersecting orbits.

The study of differentiable equivalence classes is tackled in Section 3 (simple and multiple actions), whereas Section 4 is devoted to their intersections. In the previous Section 2, we introduce some definitions and notations.

2. Preliminaries. Let $\mathcal{M}=\mathbb{C}^{n^{2}} \times \mathbb{C}^{n \times m}$ be the differentiable manifold of pairs of matrices $\mathcal{M}=\left\{(A, B): A \in \mathbb{C}^{n^{2}}, B \in \mathbb{C}^{n \times m}\right\}$ and $\mathcal{M}^{*}$ be the open dense subset of $\mathcal{M}$ formed by the controllable pairs with $\operatorname{rank} B=m$, that is to say, the full rank controllable pairs. When no confusion is possible, we identify the pair $(A, B)$ with the matrix $\left[\begin{array}{ll}A & B\end{array}\right] \in \mathbb{C}^{n \times(n+m)}$.

The usual block similarity (or BK-equivalence) is induced by the group action:

$$
\begin{aligned}
\mathcal{G}=\{g & \left.=\left[\begin{array}{cc}
S & 0 \\
R & T
\end{array}\right]: S \in G l_{n}, T \in G l_{m}, R \in \mathbb{C}^{m \times n}\right\} . \\
& g *(A, B)=S^{-1}(A, B)\left[\begin{array}{cc}
S & 0 \\
R & T
\end{array}\right]=\left(S^{-1} A S+S^{-1} B R, S^{-1} B T\right),
\end{aligned}
$$

so that the BK-equivalence class of a pair $(A, B)$ is the orbit

$$
O_{B K}(A, B)=\{g *(A, B): g \in \mathcal{G}\} .
$$

The actions of $S, T, R$ are called a change of state variables, a change of input variables and a feedback, respectively. In a natural way, we can also consider the subgroups relative to only some of these actions and their corresponding orbits.

Definition 2.1. Let $(A, B) \in \mathcal{M}$. We consider the following suborbits of $O_{B K}(A, B)$ defined by:

1. $O_{S T}(A, B), O_{S R}(A, B), O_{T R}(A, B)$ when $R=0, T=I_{m}, S=I_{n}$, respectively.

2. $O_{S}(A, B), O_{T}(A, B), O_{R}(A, B)$ when $R=0$ and $T=I_{m}, R=0$ and $S=I_{n}$, $S=I_{n}$ and $T=I_{m}$, respectively.

We will refer to them as the $S T$-orbit, $\ldots, R$-orbit of $(A, B)$, respectively. Or, equivalently, the orbit of $(A, B)$ with regard to the ST-action, $\ldots, R$-action. 
Our aim is to study these orbits and their intersections. It follows directly from the Closed Orbit Lemma (see for example [10]) that all the above orbits are differentiable manifolds. Their dimensions will be computed in Section 3. Concerning their intersections, we notice that the intersection of two differentiable manifolds may not be a differentiable manifold even if they are group orbits. However, in Section 4 we will see that in our case this fact follows from transversality conditions.

As we have pointed out, we restrict ourselves to the generic case of full rank controllable pairs. Several canonical forms and complete invariants are well-known with regard to block similarity (for a survey, see [12] and [13]). We will use the following $B K$-form, defined by means of the controllability indices. We write $E_{q}=$

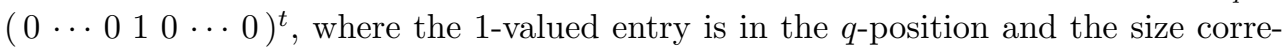
sponds to the context, and $N_{p}=\left(0, E_{1}, \ldots, E_{p-1}\right)$ is the upper nilpotent $p$-block.

Definition 2.2. Given a full rank controllable pair of matrices $(A, B) \in \mathcal{M}^{*}$ and $k_{1} \geq k_{2} \geq \cdots \geq k_{m}>0$ its controllability indices, it is known that there is another pair $\left(A_{c}, B_{c}\right)$ in its BK-orbit such that

$$
\left(A_{c}, B_{c}\right)=\left(\operatorname{diag}\left(N_{k_{1}}, N_{k_{2}}, \ldots, N_{k_{m}}\right),\left(E_{l_{1}}, E_{l_{2}}, \ldots, E_{l_{m}}\right)\right)
$$

where $l_{i}=\sum_{j=1}^{i} k_{j}$.

It is said that $\left(A_{c}, B_{c}\right)$ is the Brunovsky canonical form of $(A, B)$ or that $\left(A_{c}, B_{c}\right)$ is a $B K$-pair.

We express the pairs $(C, D) \in \mathcal{M}$ linked to a full rank controllable pair $(A, B)$ with controllability indices $k_{1} \geq k_{2} \geq \cdots \geq k_{m} \geq 0$ divided into blocks: $C=$ $\left(C_{i, j}\right)_{1 \leq i, j \leq m}, \quad C_{i, j} \in \mathbb{C}^{k_{i} \times k_{j}}, \quad D=\left(D_{1}, D_{2}, \ldots, D_{m}\right), \quad D_{i} \in \mathbb{C}^{n}$.

When 0 appears in a block matrix, it will be a null block of the suitable size (it could be empty).

The following families of parameterized matrices divided into the above blocks will be widely used in the following sections:

\section{DEFINITION 2.3.}

1. We consider $A_{\alpha}, A_{\beta} \in \mathbb{C}^{n \times n}$ and $B_{\gamma}, B_{\delta} \in \mathbb{C}^{n \times m}$ with $\alpha_{i, j, p}, \beta_{i, j, p}, \gamma_{i, j}, \delta_{i, j, l} \in$ $\mathbb{C}$ such that

$A_{\alpha_{i, j}}=E_{k_{i}} \cdot\left(\alpha_{i, j, 1}, \alpha_{i, j, 2}, \ldots, \alpha_{i, j, \min \left\{k_{i}, k_{j}\right\}}, 0\right)$,

$A_{\beta, i, j}=E_{k_{i}} \cdot\left(0, \beta_{i, j, k_{j}-k_{i}+1}, \ldots, \beta_{i, j, k_{j}}\right)$ if $k_{i}<k_{j}$, and $A_{\beta, i, j}=0$ otherwise, $B_{\gamma, j}=\sum_{k_{i}>k_{j}} \gamma_{i, j} E_{k_{i}}$

$B_{\delta, j}=\sum_{1 \leq i<j \leq m} \sum_{1 \leq l \leq k_{i, j}} \delta_{i, j, l} E_{k_{i}-l}$, where $k_{i, j}=\max \left\{0, k_{i}-k_{j}-1\right\}$.

2. Notice that $A_{\alpha}$ and $A_{\beta}$ can be seen as feedbacks $B_{c} R$. We will refer to them as $\alpha$-feedbacks and $\beta$-feedbacks, respectively. 
3. We denote the number of parameters of $A_{\alpha}, A_{\beta}, B_{\gamma}, B_{\delta}$ by

$n_{\alpha}=\sum_{i, j=1}^{m} \min \left\{k_{i}, k_{j}\right\}=\sum_{i=1}^{m}(2 i-1) k_{i}$.

$n_{\beta}=\sum_{i, j=1}^{m} \max \left\{0, k_{i}-k_{j}\right\}$.

$n_{\gamma}=\sum_{i, j=1}^{m} \Gamma_{i, j}$, where $\Gamma_{i, j}=1 \quad$ if $\quad k_{i}>k_{j}$, and 0 otherwise.

$n_{\delta}=\sum_{i, j=1}^{m} \max \left\{0, k_{i}-k_{j}-1\right\}$.

Notice that $n_{\alpha}+n_{\beta}=n m, \quad n_{\gamma}+n_{\delta}=n_{\beta}, \quad n_{\alpha}+n_{\gamma}+n_{\delta}=n m$.

EXAMPLE 2.4. If $k_{1}=6, k_{2}=3, k_{3}=k_{4}=2$, and the pair $\left(A_{c}, B_{c}\right)$ is the corresponding Brunovsky canonical form, then $A_{c}+A_{\alpha}+A_{\beta}$ and $B_{c}+B_{\gamma}+B_{\delta}$ are, respectively,

$\left[\begin{array}{ccccccc|ccc|cc|cc}0 & 1 & 0 & 0 & 0 & 0 & 0 & 0 & 0 & 0 & 0 & 0 & 0 \\ 0 & 0 & 1 & 0 & 0 & 0 & 0 & 0 & 0 & 0 & 0 & 0 & 0 \\ 0 & 0 & 0 & 1 & 0 & 0 & 0 & 0 & 0 & 0 & 0 & 0 & 0 \\ 0 & 0 & 0 & 0 & 1 & 0 & 0 & 0 & 0 & 0 & 0 & 0 & 0 \\ 0 & 0 & 0 & 0 & 0 & 1 & 0 & 0 & 0 & 0 & 0 & 0 & 0 \\ \alpha_{1,1,1} & \alpha_{1,1,2} & \alpha_{1,1,3} & \alpha_{1,1,4} & \alpha_{1,1,5} & \alpha_{1,1,6} & \alpha_{1,2,1} & \alpha_{1,2,2} & \alpha_{1,2,3} & \alpha_{1,3,1} & \alpha_{1,3,2} & \alpha_{1,4,1} & \alpha_{1,4,2} \\ \hline 0 & 0 & 0 & 0 & 0 & 0 & 0 & 1 & 0 & 0 & 0 & 0 & 0 \\ 0 & 0 & 0 & 0 & 0 & 0 & 0 & 0 & 1 & 0 & 0 & 0 & 0 \\ \alpha_{2,1,1} & \alpha_{2,1,2} & \alpha_{2,1,3} & \beta_{2,1,4} & \beta_{2,1,5} & \beta_{2,1,6} & \alpha_{2,2,1} & \alpha_{2,2,2} & \alpha_{2,2,3} & \alpha_{2,3,1} & \alpha_{2,3,2} & \alpha_{2,4,1} & \alpha_{2,4,2} \\ \hline 0 & 0 & 0 & 0 & 0 & 0 & 0 & 0 & 0 & 0 & 1 & 0 & 0 \\ \alpha_{3,1,1} & \alpha_{3,1,2} & \beta_{3,1,3} & \beta_{3,1,4} & \beta_{3,1,5} & \beta_{3,1,6} & \alpha_{3,2,1} & \alpha_{3,2,2} & \beta_{3,2,3} & \alpha_{3,3,1} & \alpha_{3,3,2} & \alpha_{3,4,1} & \alpha_{3,4,2} \\ \hline 0 & 0 & 0 & 0 & 0 & 0 & 0 & 0 & 0 & 0 & 0 & 0 & 1 \\ \alpha_{4,1,1} & \alpha_{4,1,2} & \beta_{4,1,3} & \beta_{4,1,4} & \beta_{4,1,5} & \beta_{4,1,6} & \alpha_{4,2,1} & \alpha_{4,2,2} & \beta_{4,2,3} & \alpha_{4,3,1} & \alpha_{4,3,2} & \alpha_{4,4,1} & \alpha_{4,4,2}\end{array}\right]$

$\left[\begin{array}{c|c|c|c}0 & 0 & 0 & 0 \\ 0 & 0 & 0 & 0 \\ 0 & 0 & \delta_{1,3,3} & \delta_{1,4,3} \\ 0 & \delta_{1,2,2} & \delta_{1,3,2} & \delta_{1,4,2} \\ 0 & \delta_{1,2,1} & \delta_{1,3,1} & \delta_{1,4,1} \\ 1 & \gamma_{1,2} & \gamma_{1,3} & \gamma_{1,4} \\ \hline 0 & 0 & 0 & 0 \\ 0 & 0 & 0 & 0 \\ 0 & 1 & \gamma_{2,3} & \gamma_{2,4} \\ \hline 0 & 0 & 0 & 0 \\ 0 & 0 & 1 & 0 \\ \hline 0 & 0 & 0 & 0 \\ 0 & 0 & 0 & 1\end{array}\right]$

In what follows, the linear space of pairs $\left\{\left(A_{\alpha}, 0\right), \alpha \in \mathbb{C}^{n_{\alpha}}\right\}$ will be simply denoted by $\left\{\left(A_{\alpha}, 0\right)\right\}_{\alpha}$; and similarly for $\left\{\left(A_{\beta}, 0\right)\right\}_{\beta},\left\{\left(0, B_{\delta}\right)\right\}_{\delta}$ and $\left\{\left(0, B_{\gamma}\right)\right\}_{\gamma}$.

3. The orbits $O_{S}, O_{T}, O_{R}, O_{B K}, O_{S T}$, and $O_{S R}$. It is easy to prove that, for full rank controllable pairs, the single actions $S, T$, and $R$ generate orbits of constant 
dimension.

Proposition 3.1. Let $(A, B) \in \mathcal{M}$.

1. If $(A, B)$ is controllable, $\operatorname{dim} O_{S}(A, B)=n^{2}$.

2. If $B$ has full rank, $\operatorname{dim} O_{R}(A, B)=n m$.

3. If $B$ has full rank, $\operatorname{dim} O_{T}(A, B)=m^{2}$.

Proof. In general (see, for example, [3, Section I.1]) if a group $\mathcal{G}$ acts on a manifold $\mathcal{M}$, the dimension of the orbit $O_{\mathcal{G}}(x)$ of a point $x \in \mathcal{M}$ is the codimension of the stabilizer subgroup $\{g \in \mathcal{G}: g * x=x\}$. In [8] and [15] (Part IV) one proves that the pair is controllable if and only if the stabilizer with regard to the $S$-action is trivial. Next, we conclude it from the fact that the differential (at the identity element) of the action $g \longrightarrow g * x$ is injective. This argument will also work for assertions 2 and 3 .

For the $(S, T, R)$-action we have

$$
\begin{gathered}
\varphi: \mathbb{C}^{n^{2}} \times \mathbb{C}^{m^{2}} \times \mathbb{C}^{m \times n} \longrightarrow \mathcal{M}, \quad(S, T, R) \longmapsto\left(S^{-1} A S+S^{-1} B R, S^{-1} B T\right) ; \\
D \varphi_{\left(I d_{n}, I d_{m}, 0\right)}\left(S^{\prime}, T^{\prime}, R^{\prime}\right)=\left(A S^{\prime}-S^{\prime} A+B R^{\prime}, B T^{\prime}-S^{\prime} B\right) .
\end{gathered}
$$

In particular, if only $S$ varies,

$$
\frac{\partial \varphi}{\partial S}_{\left(I d_{n}, I d_{m}, 0\right)}\left(S^{\prime}\right)=\left(A S^{\prime}-S^{\prime} A,-S^{\prime} B\right)
$$

which is injective if the pair $(A, B)$ is controllable: $A S^{\prime}=S^{\prime} A$ and $S^{\prime} B=0$ implies $S^{\prime}\left(B, A B, A_{2} B, \ldots, A_{n-1} B\right)=0$ and, because of the controllability of $(A, B), S^{\prime}=0$.

Analogously,

$$
\frac{\partial \varphi}{\partial R}_{\left(I d_{n}, I d_{m}, 0\right)}\left(R^{\prime}\right)=\left(B R^{\prime}, 0\right), \quad \frac{\partial \varphi}{\partial T}_{\left(I d_{n}, I d_{m}, 0\right)}\left(T^{\prime}\right)=\left(0, B T^{\prime}\right)
$$

are injective if $B$ has full column rank.

As we have pointed out, we are interested in the intersections of the above orbits and the mixed actions. In fact, we focus on the cases when the $S$-action is involved because of the trivial fact that:

Proposition 3.2. Let $(A, B) \in \mathcal{M}^{*}$ be a full rank controllable pair of matrices. Then

1. $O_{T}(A, B) \cap O_{R}(A, B)=\{(A, B)\}$.

2. $\operatorname{dim} O_{T R}(A, B)=\operatorname{dim} O_{T}(A, B)+\operatorname{dim} O_{R}(A, B)=m^{2}+n m$. 
We recall that the triple action of $S, T$ and $R$ corresponds to the usual block similarity. The geometric structure of the $B K$-orbits has been studied in [3] and [4] by means of Arnold's techniques introduced in [2]. See, for example, [3, Sections (I.2) and (II.1)] for the basic definitions and results concerning versal/miniversal deformations. We will refer to $B K$-miniversal, $S$-miniversal, ...the miniversal deformations with regard to the $B K$-action, $S$-action, ... In particular, for a full rank controllable pair, we have:

TheOREM 3.3. [3] Given a full rank controllable pair $(A, B) \in \mathcal{M}^{*}$ with controllability indices $k_{1} \geq k_{2} \geq \cdots \geq k_{m}>0$, then

$$
\operatorname{dim} O_{B K}(A, B)=n^{2}+n m-n_{\delta}=n^{2}+n_{\alpha}+n_{\gamma}
$$

If $\left(A_{c}, B_{c}\right)$ is its Brunovsky canonical form, a BK-miniversal deformation of $\left(A_{c}, B_{c}\right)$ in $\mathcal{M}^{*}$ is the $n_{\delta}$-dimensional linear manifold $\left(A_{c}, B_{c}\right)+\left\{\left(0, B_{\delta}\right)\right\}_{\delta}$.

Canonical forms with regard to the change of states have been obtained for controllable pairs by several authors $([1],[7],[9],[11],[14],[15])$. In fact, we will base the study of the orbits $O_{S T}$ and $O_{S R}$ on the following result, which is a direct consequence of Theorems (2.2) and (2.3) in [15], jointly with the above Theorem 3.3 and (1) of Proposition 3.1:

TheOREM 3.4. Let $(A, B) \in \mathcal{M}^{*}$ be a full rank controllable pair with controllability indices $k_{1} \geq k_{2} \geq \cdots \geq k_{m}>0$, and $\left(A_{c}, B_{c}\right)$ its Brunovsky canonical form. Then:

An $S$-miniversal deformation of $\left(A_{c}, B_{c}\right)$ in its BK-orbit is given by the $\left(n_{\alpha}+n_{\gamma}\right)$ dimensional linear manifold $\left(A_{c}, B_{c}\right)+\left\{\left(A_{\alpha}, B_{\gamma}\right)\right\}_{\alpha, \gamma}$.

This $S$-miniversal deformation of $\left(A_{c}, B_{c}\right)$ has the following property: the $\gamma$ parameters, and only they, can be eliminated by the $T$-action; and analogously, the $\alpha$-parameters, and only they, by the $R$-action. Therefore:

Proposition 3.5. In the conditions of Theorem 3.4:

1. The $n_{\alpha}$-dimensional linear manifold $\left(A_{c}, B_{c}\right)+\left\{\left(A_{\alpha}, 0\right)\right\}_{\alpha} \subset O_{S R}\left(A_{c}, B_{c}\right)$ is an ST-miniversal deformation of $\left(A_{c}, B_{c}\right)$ in its BK-orbit.

2. The $n_{\gamma}$-dimensional linear manifold $\left(A_{c}, B_{c}\right)+\left\{\left(0, B_{\gamma}\right)\right\}_{\gamma} \subset O_{S T}\left(A_{c}, B_{c}\right)$ is an SR-miniversal deformation of $\left(A_{c}, B_{c}\right)$ in its BK-orbit.

As a first direct consequence of this result, we have:

Corollary 3.6. Given a full rank controllable BK-pair $\left(A_{c}, B_{c}\right) \in \mathcal{M}^{*}$, we have $\operatorname{dim} O_{S T}\left(A_{c}, B_{c}\right)=n^{2}+n_{\gamma}, \quad \operatorname{dim} O_{S R}\left(A_{c}, B_{c}\right)=n^{2}+n_{\alpha}$. 
EXAmple 3.7. Let us consider $n=5$ and $m=2$. Then, there are only two kinds of orbits, according to the controllability indices being $(4,1)$ or $(3,2)$ (see Table 3.1 ). Let us obtain the bifurcation diagram in Figure 3.1, that is to say, the partition in the $\left(\delta_{1}, \delta_{2}, \gamma\right)$-space induced by the partition of the space of pairs of matrices into the considered orbits (in our case, the $B K$-orbits, and the $S T$ and $S R$-suborbits).

\begin{tabular}{|c|c|c|c|c|c|c|c|}
\hline$k$ & $n_{\alpha}$ & $n_{\gamma}$ & $n_{\delta}$ & $\operatorname{codim} O_{B K}$ & $\operatorname{codim} O_{S T}$ & $\operatorname{codim} O_{S R}$ & $\operatorname{codim} O_{S}$ \\
\hline 4,1 & 7 & 1 & 2 & 2 & 9 & 3 & 10 \\
3,2 & 9 & 1 & 0 & 0 & 9 & 1 & 10 \\
\hline
\end{tabular}

The orbits $O_{B K}, O_{S T}$, and $O_{S R}$.

An $S R$-miniversal deformation in $\mathcal{M}^{*}$ of the pair

$$
\left(A_{c}, B_{c}\right)=\left(\left[\begin{array}{llll|l}
0 & 1 & 0 & 0 & 0 \\
0 & 0 & 1 & 0 & 0 \\
0 & 0 & 0 & 1 & 0 \\
0 & 0 & 0 & 0 & 0 \\
\hline 0 & 0 & 0 & 0 & 0
\end{array}\right],\left[\begin{array}{l|l}
0 & 0 \\
0 & 0 \\
0 & 0 \\
1 & 0 \\
\hline 0 & 1
\end{array}\right]\right) \in O_{B K}(4,1)
$$

is the 3-dimensional linear manifold formed by the pairs

$$
\left(A_{c}, B_{c}\left(\delta_{1}, \delta_{2}, \gamma\right)\right)=\left(\left[\begin{array}{cccc|c}
0 & 1 & 0 & 0 & 0 \\
0 & 0 & 1 & 0 & 0 \\
0 & 0 & 0 & 1 & 0 \\
0 & 0 & 0 & 0 & 0 \\
\hline 0 & 0 & 0 & 0 & 0
\end{array}\right],\left[\begin{array}{c|c}
0 & 0 \\
0 & \delta_{1} \\
0 & \delta_{2} \\
1 & \gamma \\
\hline 0 & 1
\end{array}\right]\right)
$$

Here, the orbit $O_{B K}(4,1)$ appears as the $\gamma$-axis, formed by pairs belonging to different SR-orbits of type $k=(4,1)$, parameterized by $\gamma$.

The remaining points correspond to the orbit $O_{B K}(3,2)$. In particular, the points in the plane $\left(\delta_{1}, \delta_{2}\right)$, or equivalently $\gamma=0$, with $\left(\delta_{1}, \delta_{2}\right) \neq(0,0)$ can be S-transformed according to Theorem 3.4. A quite laborious computation shows that

- If $\delta_{2} \neq 0$, then $\left(A_{c}, B_{c}\left(\delta_{1}, \delta_{2}, 0\right)\right)$ is $S$-equivalent to

$$
\left(\left[\begin{array}{ccc|cc}
0 & 1 & 0 & 0 & 0 \\
0 & 0 & 1 & 0 & 0 \\
0 & 0 & 0 & 0 & 1 / \delta_{2} \\
\hline 0 & 0 & 0 & 0 & 1 \\
0 & 0 & 0 & 0 & 0
\end{array}\right],\left[\begin{array}{c|c}
0 & 0 \\
0 & 0 \\
-\delta_{1} / \delta_{2} 2 & 1 \\
\hline 0 & 0 \\
1 & 0
\end{array}\right]\right) \in O_{B K}(3,2) .
$$


- If $\delta_{2}=0$ and $\delta_{1} \neq 0$, then $\left(A_{c}, B_{c}\left(\delta_{1}, \delta_{2}, 0\right)\right)$ is $S$-equivalent to

$$
\left(\left[\begin{array}{ccc|cc}
0 & 1 & 0 & 0 & 0 \\
0 & 0 & 1 & 0 & 0 \\
0 & 0 & 0 & 0 & 0 \\
\hline 0 & 0 & 0 & 0 & 1 \\
0 & 1 / \delta_{1} & 0 & 0 & 0
\end{array}\right],\left[\begin{array}{l|l}
0 & 0 \\
0 & 0 \\
1 & 0 \\
\hline 0 & 0 \\
0 & 1
\end{array}\right]\right) \in O_{B K}(3,2) .
$$

Each one lies in a different ST-orbit, because they correspond to different values of the $\alpha$-parameters.

Since the SR-orbits correspond to the $\gamma$-parameters, they are the axes $\delta_{2} \neq 0$, $\delta_{1}=0$, and $\delta_{1} \neq 0, \delta_{2}=0$, and the parabolas $\frac{\delta_{1}}{\delta_{2}^{2}}=$ constant, $\delta_{2} \neq 0$.

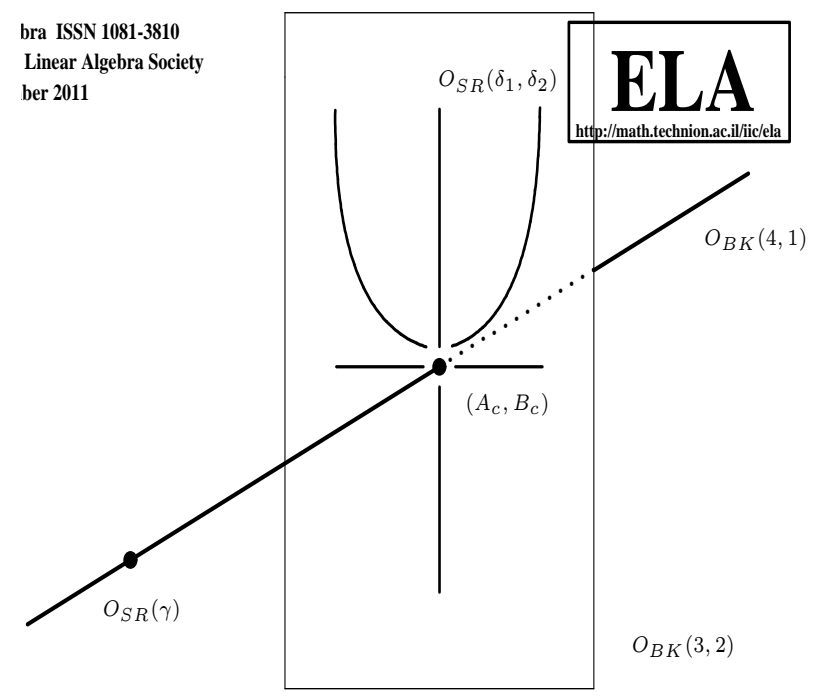

FIG. 3.1. The orbits $O_{B K}, O_{S T}$, and $O_{S R}$.

4. The intersections $O_{S} \cap O_{T}, O_{R} \cap O_{S}$, and $O_{S R} \cap O_{S T}$. Next we study the intersection of the orbits in Section 3. As we have pointed out, an orbit intersection may not be an orbit, not even a differentiable manifold. However, in our case we have:

Proposition 4.1. Let $(A, B) \in \mathcal{M}^{*}$ be a full rank controllable pair with controllability indices $k_{1} \geq \cdots \geq k_{m}>0$. Then the orbit intersections $O_{S} \cap O_{T}$ and $O_{S} \cap O_{R}$ are differentiable submanifolds of $O_{B K}(A, B)$ and:

$$
\begin{aligned}
& \operatorname{dim}\left(O_{S}(A, B) \cap O_{T}(A, B)\right)=n^{2}+m^{2}-\operatorname{dim} O_{S T}(A, B), \\
& \operatorname{dim}\left(O_{S}(A, B) \cap O_{R}(A, B)\right)=n^{2}+n m-\operatorname{dim} O_{S R}(A, B) .
\end{aligned}
$$


If $(A, B)$ is a BK-pair, the dimensions of the above intersections are $m^{2}-n_{\gamma}$ and $n m-n_{\alpha}$, respectively.

Proof. It is well known (see, for example, [6, page 30]) that if two submanifolds (of a manifold) are transversal, then their intersection is also a submanifold and its codimension is the sum of the codimensions of the given ones.

In our case, $O_{S}(A, B)$ and $O_{T}(A, B)$ are transversal in $O_{S T}(A, B)$ at $(A, B)$ because the tangent space

$$
T_{(A, B)} O_{S T}(A, B)=\left\{\left(A S^{\prime}-S^{\prime} A, B T^{\prime}-S^{\prime} B\right) ; S^{\prime} \in \mathbb{C}^{n^{2}}, T^{\prime} \in \mathbb{C}^{m^{2}}\right\}
$$

(see the proof of Proposition 3.1) is clearly spanned by the analogously described $T_{(A, B)} O_{S}(A, B)$ and $T_{(A, B)} O_{T}(A, B)$.

Therefore, their intersection is a submanifold of $O_{S T}(A, B)$ (and hence also of $\left.O_{B K}(A, B)\right)$ with dimension:

$$
\operatorname{dim}\left(O_{S}(A, B) \cap O_{T}(A, B)\right)=\operatorname{dim} O_{S}(A, B)+\operatorname{dim} O_{T}(A, B)-\operatorname{dim} O_{S T}(A, B)
$$

and the results follow from Proposition 3.1 and Corollary 3.6.

Analogously for the second assertion.

Given two subgroups $\mathcal{G}_{1}$ and $\mathcal{G}_{2}$ of a group $\mathcal{G}$, the intersection of their orbits may not be the orbit corresponding to $\mathcal{G}_{1} \cap \mathcal{G}_{2}$. For example, the intersection of the $S$-subgroup and the $T$-subgroup is simply the identity matrix. It may not even be an orbit. However, let us see that the intersections in Proposition 4.1 are indeed orbits with regard to the action of suitable subgroups which will depend on the pair $(A, B)$. We will describe it explicitly when $(A, B)$ is a $B K$-pair.

For the first intersection this description is obtained by means of the following class of Toeplitz matrices:

\section{DeFinition 4.2.}

(1) $X \in \mathbb{C}^{p \times q}$ is called an upper triangular Toeplitz matrix if

(i) It is constant along the diagonals $x_{i, j}=x_{i+1, j+1}$,

(ii) $x_{i, 1}=0$ if $i>1$,

(iii) $x_{1, i}=0$ if $i \leq q-p$.

(2) If $k_{1}, \ldots, k_{m}$ is a partition of $n$ (that is, $k_{1}+\cdots+k_{m}=n$ ), $X \in \mathbb{C}^{n \times n}$ is called a block upper triangular Toeplitz matrix if

$$
X=\left(X_{i, j}\right)_{1 \leq i, j \leq m}, \quad X_{i, j} \in \mathbb{C}^{k_{i} \times k_{j}}
$$

and each $X_{i, j}$ is an upper triangular Toeplitz matrix. We will denote the set of these matrices by $U T T\left(k_{1}, \ldots, k_{m}\right)$. 
We recall also that if $A$ is a block diagonal nilpotent matrix

$$
A=\operatorname{diag}\left(N_{k_{1}}, \ldots, N_{k_{m}}\right)
$$

then a nonsingular matrix $S \in G l_{n}$ belongs to its centralizer (that is: $S^{-1} A S=A$ ) if and only if $S \in U T T\left(k_{1}, \ldots, k_{m}\right)$.

With this notation, we have:

Proposition 4.3. Let $(A, B) \in \mathcal{M}^{*}$ be a full rank controllable pair.

(1) The submanifold $O_{S}(A, B) \cap O_{T}(A, B)$ is the orbit of $(A, B)$ with regard to the action of $\mathcal{G}_{S T}(A, B)$ formed by the $S \in G l_{n}$ such that $A S=S A$, and there is $T \in G l_{m}$ such that: $S B=B T$.

(2) In particular, if $\left(A_{c}, B_{c}\right) \in \mathcal{M}^{*}$ is a BK-pair with controllability indices $k_{1} \geq$ $\cdots \geq k_{m}>0$, then:

$$
\begin{gathered}
\mathcal{G}_{S T}\left(A_{c}, B_{c}\right)=\left\{S \in G l_{n} \cap U T T\left(k_{1}, \ldots, k_{m}\right): S_{i, j}=0 \quad \text { if } \quad k_{i}>k_{j},\right. \\
\text { and } \left.S_{i, j}=\left(0, s_{i, j} I_{k_{i}}\right) \text { if } \quad k_{i} \leq k_{j}\right\} .
\end{gathered}
$$

Proof.

(1) It is obvious that $O_{T}(A, B) \cap O_{S}(A, B)=\left\{(A, S B): S \in \mathcal{G}_{S T}\right\}$. Hence, it is sufficient to check that $\mathcal{G}_{S T}$ is a subgroup of the centralizer of $A$ : if for $i=1,2, S_{i} \in \mathcal{G}_{S T}$ and $T_{i} \in G l_{m}$ are such that $S_{i} B=B T_{i}$, then left multiplying by $S_{i}^{-1}$ and right multiplying by $T_{i}^{-1}$ we have $S_{i}^{-1} B=B T_{i}^{-1}$ and $S_{1} S_{2}^{-1} B=S_{1} B T_{2}^{-1}=B T_{1} T_{2}^{-1}$.

(2) We have noticed that the set of nonsingular matrices $S$ such that $A S=S A$ is $\operatorname{UTT}\left(k_{1}, \ldots, k_{m}\right)$.

On the other hand, if $B_{c}=\left(E_{l_{1}}, E_{l_{2}}, \ldots, E_{l_{m}}\right)$, the columns of $S B_{c}$ will be the columns $l_{1}, l_{2}, \ldots, l_{m}$ of $S$. Moreover, because $S B_{c}=B_{c} T$, these columns must be linear combinations of the columns of $B_{c}$. Hence, $\left(S_{i, j}\right)_{t, k_{j}}=0$ if $1 \leq t<k_{i}$, and the proposition is proved.

Concerning the second intersection in Proposition 4.1, we have again a general description as an orbit with regard to a group depending on the pair $(A, B)$, and an explicit description for $B K$-pairs $\left(A_{c}, B_{c}\right)$ :

TheOrem 4.4. Let $(A, B) \in \mathcal{M}^{*}$ be a full rank controllable pair.

(1) The submanifold $O_{S}(A, B) \cap O_{R}(A, B)$ is the orbit of $(A, B)$ with regard to the action of $\mathcal{G}_{S R}(A, B)$ formed by the $S \in G l_{n}$ such that $S^{-1} B=B$, and there is $R \in \mathbb{C}^{m \times n}$ such that: $S^{-1} A S=A+B R$. 
(2) In particular, if $\left(A_{c}, B_{c}\right) \in \mathcal{M}^{*}$ is a BK-pair with controllability indices $k_{1} \geq$ $\cdots \geq k_{m}>0$, it is a $n_{\beta}$-dimensional linear manifold:

$$
O_{S}\left(A_{c}, B_{c}\right) \cap O_{R}\left(A_{c}, B_{c}\right)=\left(A_{c}, B_{c}\right)+\left\{\left(A_{\beta}, 0\right)\right\}_{\beta}
$$

Proof.

(1) It is obvious that:

$$
O_{S}(A, B) \cap O_{R}(A, B)=\left\{\left(S^{-1} A S, B\right): S \in \mathcal{G}_{S R}(A, B)\right\}
$$

Hence, it is sufficient to check that $\mathcal{G}_{S R}$ is a subgroup of $G l_{n}$ : if for $i=1,2$ one has $S_{i}^{-1} A S_{i}=A+B R_{i}$ and $S_{i}^{-1} B=B$, then

$$
\begin{aligned}
\left(S_{1} S_{2}^{-1}\right)^{-1} B & =S_{2} S_{1}^{-1} B=S_{2} B=B \\
\left(S_{1} S_{2}^{-1}\right)^{-1} A\left(S_{1} S_{2}^{-1}\right) & =S_{2}\left(A+B R_{1}\right) S_{2}^{-1}= \\
A+B R_{2}+S_{2} B R_{1} S_{2}^{-1} & =A+B\left(R_{2}+R_{1} S_{2}^{-1}\right)
\end{aligned}
$$

(2) For simplicity we will refer to the given pair as $(A, B)$, to its orbits as $O_{R}$ and $O_{S}$ and to the $n_{\beta}$-linear manifold as $L_{\beta}$.

Firstly, we prove that $L_{\beta} \subset O_{R}$ : taking the rows $R^{j}=A_{\beta}^{l_{j}}$ and bearing in mind that $B^{l_{j}}=E_{j}^{T}$ and $B^{i}=0$ otherwise, it is easy to see that $A_{\beta}=B R$.

Secondly, to prove that $L_{\beta} \subset O_{S}$ we must see that there is $X \in G l_{n}$ such that $X A X^{-1}=A+A_{\beta}$ and $X B=B$ or, equivalently, $X A=A X+A_{\beta} X$ and $X B=B$.

Expressing these conditions in blocks, we have

(a) $X_{i, j} N_{k_{j}}=N_{k_{i}} X_{i, j}+\sum_{p=1}^{i-1} A_{\beta, i, p} X_{p, j}$,

(b) $\left(X_{i, j}\right)_{k_{j}}=\delta_{i, j} E_{k_{i}}$.

We define

(a) $S \in G l_{n}$ such that $S_{i, i}=I_{k_{i}}, S_{i, j}=0$ if $k_{i} \geq k_{j}$ and $\left(S_{i, j}\right)^{p}=$ $\sum_{q=1}^{k_{j}-k_{i}} \beta_{i, j, q} E_{p+q-1}^{T}$ if $k_{i}<k_{j}$.

(b) $X \in G l_{n}$ such that $X_{i, i}=I_{k_{i}}, X_{i, j}=0$ if $i<j, X_{i, i-1}=S_{i, i-1}$ and $X_{i, j}=\sum_{p=i-1}^{j} S_{i, p} X_{p, j}$ if $i>j$. 
In Example 2.4, the matrix $S$ is

$\left[\begin{array}{cccccc|ccc|cc|cc}1 & 0 & 0 & 0 & 0 & 0 & 0 & 0 & 0 & 0 & 0 & 0 & 0 \\ 0 & 1 & 0 & 0 & 0 & 0 & 0 & 0 & 0 & 0 & 0 & 0 & 0 \\ 0 & 0 & 1 & 0 & 0 & 0 & 0 & 0 & 0 & 0 & 0 & 0 & 0 \\ 0 & 0 & 0 & 1 & 0 & 0 & 0 & 0 & 0 & 0 & 0 & 0 & 0 \\ 0 & 0 & 0 & 0 & 1 & 0 & 0 & 0 & 0 & 0 & 0 & 0 & 0 \\ 0 & 0 & 0 & 0 & 0 & 1 & 0 & 0 & 0 & 0 & 0 & 0 & 0 \\ \hline \beta_{2,1,4} & \beta_{2,1,5} & \beta_{2,1,6} & 0 & 0 & 0 & 1 & 0 & 0 & 0 & 0 & 0 & 0 \\ 0 & \beta_{2,1,4} & \beta_{2,1,5} & \beta_{2,1,6} & 0 & 0 & 0 & 1 & 0 & 0 & 0 & 0 & 0 \\ 0 & 0 & \beta_{2,1,4} & \beta_{2,1,5} & \beta_{2,1,6} & 0 & 0 & 0 & 1 & 0 & 0 & 0 & 0 \\ \hline \beta_{3,1,3} & \beta_{3,1,4} & \beta_{3,1,5} & \beta_{3,1,6} & 0 & 0 & \beta_{3,2,3} & 0 & 0 & 1 & 0 & 0 & 0 \\ 0 & \beta_{3,1,3} & \beta_{3,1,4} & \beta_{3,1,5} & \beta_{3,1,6} & 0 & 0 & \beta_{3,2,3} & 0 & 0 & 1 & 0 & 0 \\ \hline \beta_{4,1,3} & \beta_{4,1,4} & \beta_{4,1,5} & \beta_{4,1,6} & 0 & 0 & \beta_{4,2,3} & 0 & 0 & 0 & 0 & 1 & 0 \\ 0 & \beta_{4,1,3} & \beta_{4,1,4} & \beta_{4,1,5} & \beta_{4,1,6} & 0 & 0 & \beta_{4,2,3} & 0 & 0 & 0 & 0 & 1\end{array}\right]$

Notice that obviously $S B=B$. In addition, if $i>j$, we have

- $S_{i, j} A_{\beta, j, p}=0$.

- $S_{i, j} N_{k_{j}}=N_{k_{i}} S_{i, j}+A_{\beta, i, j}$.

- $X_{i, j} N_{k_{j}}=\sum_{p=i-1}^{j} S_{i, p} X_{p, j} N_{k_{j}}=\sum_{p=i-1}^{j} S_{i, p} N_{k_{p}} X_{p, j}=$ $\sum_{p=i-1}^{j}\left(N_{k_{i}} S_{i, p}+A_{\beta, i, p}\right) X_{p, j}=N_{k_{i}} X_{i, j}+\sum_{p=i-1}^{j} A_{\beta, i, p} X_{p, j}=$ $N_{k_{i}} X_{i, j}+\sum_{p=1}^{i-1} A_{\beta, i, p} X_{p, j}$.

- $\left(X_{i, i}\right)_{k_{i}}=E_{k_{i}},\left(X_{i, i-1}\right)_{k_{i-1}}=\left(S_{i, i-1}\right)_{k_{i-1}}=0$ and by recurrence $\left(X_{i, j}\right)_{k_{j}}=\delta_{i, j} E_{k_{i}}$.

The last equalities prove that $L_{\beta} \subset O_{S}$. Then, $L_{\beta} \subset O_{S} \cap O_{R}$. The two manifolds have the same dimension $n_{\beta}$. Let us see that the above inclusion is an equality. It is a straightforward computation that if a pair $\left(A_{0}, B_{0}\right)=$ $(A, B)+\left(A_{\alpha}, 0\right)+\left(A_{\beta}, 0\right) \in O_{R}(A, B)$ belongs to $O_{S}(A, B)$, then $\alpha=0$ and hence it lies in $L_{\beta}$; the condition $\operatorname{rank} A_{0}=\operatorname{rank} A$, implies that the $\alpha$-parameters in the first column of each block (i.e., in the columns $1, q_{1}+1$, $\left.q_{2}+1, \ldots\right)$ must be zero; then, the condition $\operatorname{rank} A_{0}^{2}=\operatorname{rank} A_{c}^{2}$ implies that the $\alpha$-parameters in the second column of each block (i.e., in the columns 2 , $\left.q_{1}+2, \ldots\right)$ must be zero; and so on.

\section{REMARK 4.5 .}

1. It is well known that, if $(A, B)$ is controllable, the eigenvalues of $A$ can be shifted by means of suitable feedbacks. Even more, Rosenbrock's theorem details the effects of feedbacks on the Jordan form of $A$. The last assertion in Theorem 4.4 shows that in fact the $\beta$-feedbacks do not change the Jordan invariants of $A_{c}$. On the other hand, Arnold's theory shows that $A_{c}+\left\{A_{\alpha}\right\}_{\alpha}$ is a Jordan-miniversal deformation of $A_{c}$, so that any nonzero $\alpha$-feedback of $\left(A_{c}, B_{c}\right)$ modifies the Jordan invariants of $A_{c}$. 
2. More explicitly, in the above proof one has described, for each $\beta$-feedback, the change of basis $S_{\beta} \in G l_{n}$ such that

$$
S_{\beta}^{-1} A_{c} S_{\beta}=A_{c}+A_{\beta}=A_{c}+B_{c} R_{\beta}, \quad S_{\beta}^{-1} B_{c}=B_{c}
$$

It gives an alternative explicit description of $O_{S} \cap O_{R}$ :

$$
O_{S}\left(A_{c}, B_{c}\right) \cap O_{R}\left(A_{c}, B_{c}\right)=\left(A_{c}, B_{c}\right)+\left\{\left(A_{\beta}, 0\right)\right\}_{\beta}=\left\{\left(S_{\beta}^{-1} A_{c} S_{\beta}, B_{c}\right)\right\}_{\beta} .
$$

As above, let us see that $O_{S R}(A, B) \cap O_{S T}(A, B)$ is a differentiable submanifold of $O_{B K}(A, B)$. Notice that obviously $O_{S}(A, B) \subset O_{S R}(A, B) \cap O_{S T}(A, B)$. We will see that in fact the converse is also true if $(A, B)$ is a $B K$-pair. Previously we prove a similar result concerning $O_{R}\left(A_{c}, B_{c}\right) \cap O_{S T}\left(A_{c}, B_{c}\right)$ :

Lemma 4.6. Let $(A, B) \in \mathcal{M}^{*}$ be a full rank controllable pair.

(1) The intersection $O_{S T}(A, B) \cap O_{R}(A, B)$ is a submanifold of $O_{B K}(A, B)$.

(2) In particular, if $\left(A_{c}, B_{c}\right) \in \mathcal{M}^{*}$ is a BK-pair,

$\left(A_{c}, B_{c}\right)+\left\{\left(A_{\beta}, 0\right)\right\}_{\beta}=O_{S}\left(A_{c}, B_{c}\right) \cap O_{R}\left(A_{c}, B_{c}\right)=O_{S T}\left(A_{c}, B_{c}\right) \cap O_{R}\left(A_{c}, B_{c}\right)$.

Proof.

(1) As in Proposition 4.1.

(2) With the notation in the proof of Theorem 4.4 (2), we have shown that:

$$
L_{\beta}=O_{S} \cap O_{R}\left(A_{c}, B_{c}\right) \subset O_{S T}\left(A_{c}, B_{c}\right) \cap O_{R}\left(A_{c}, B_{c}\right) .
$$

If $\left(A^{\prime}, B^{\prime}\right) \in O_{S T}\left(A_{c}, B_{c}\right) \cap O_{R}\left(A_{c}, B_{c}\right)$, then:

$$
A^{\prime}=S^{-1} A S=A+A_{\alpha}+A_{\beta}, \quad B^{\prime}=S^{-1} B T=B
$$

for some $S, T, \alpha, \beta$. We have also proved that the first relation implies $\alpha=0$, and that then (see Remark 4.5):

$$
A+A_{\beta}=S_{\beta}^{-1} A S_{\beta}, \quad B=S_{\beta}^{-1} B
$$

Hence, $\left(A^{\prime}, B^{\prime}\right) \in O_{S} \cap O_{R} . \quad \square$

TheOREM 4.7. $\operatorname{Let}(A, B) \in \mathcal{M}^{*}$ be a full rank controllable pair.

(1) The intersection $O_{S R}(A, B) \cap O_{S T}(A, B)$ is a differentiable submanifold of $O_{B K}(A, B)$ and

$$
\begin{gathered}
n^{2} \leq \operatorname{dim}\left(O_{S R}(A, B) \cap O_{S T}(A, B)\right)= \\
\operatorname{dim} O_{S R}(A, B)+\operatorname{dim} O_{S T}(A, B)-\left(n^{2}+n_{\alpha}+n_{\gamma}\right) .
\end{gathered}
$$


(2) In particular, if $\left(A_{c}, B_{c}\right) \in \mathcal{M}^{*}$ is a BK-pair, then

$$
O_{S R}\left(A_{c}, B_{c}\right) \cap O_{S T}\left(A_{c}, B_{c}\right)=O_{S}\left(A_{c}, B_{c}\right) .
$$

Proof.

(1) As in Proposition 4.1.

(2) For $B K$-pairs, we have $\operatorname{dim}\left(O_{S R}(A, B) \cap O_{S T}(A, B)\right)=n^{2}=\operatorname{dim} O_{S}(A, B)$. Again we refer to the given pair simply as $(A, B)$. If $\left(A^{\prime}, B^{\prime}\right)$ lies in the intersection, there will be $S_{1}, S_{2}, R$ and $T$ such that:

$$
A^{\prime}=S_{1}^{-1} A S_{1}=S_{2}^{-1}(A+B R) S_{2}, B^{\prime}=S_{1}^{-1} B T=S_{2}^{-1} B .
$$

Let $A^{\prime \prime}=S_{2} A^{\prime} S_{2}^{-1}=S_{3}^{-1} A S_{3}=A+B R$, where $S_{3}=S_{1} S_{2}^{-1}$. Then, $B=S_{2} B^{\prime}=S_{3}^{-1} B T$. Clearly

$$
\begin{gathered}
\left(A^{\prime \prime}, B\right) \in O_{S}\left(A^{\prime}, B^{\prime}\right), \\
\left(A^{\prime \prime}, B\right) \in O_{S T}(A, B) \cap O_{R}(A, B) \supset O_{S}(A, B) \cap O_{R}(A, B) .
\end{gathered}
$$

But Lemma 4.6 ensures that the last inclusion is in fact an equality. Hence $\left(A^{\prime \prime}, B\right) \in O_{S}(A, B)$. From it and $\left(A^{\prime \prime}, B\right) \in O_{S}\left(A^{\prime}, B^{\prime}\right)$, one has $\left(A^{\prime}, B^{\prime}\right) \in$ $O_{S}(A, B)$.

REMARK 4.8. The last assertion in Theorem 4.7 shows that, in this case, the intersection $O_{S T} \cap O_{S R}$ is just the orbit generated by the action of the intersection Lie subgroup of those generating $O_{S T}$ and $O_{S R}$.

\section{Some comments and remarks.}

5.1. Summary diagram. We can summarize the above results in the way shown in Figure 5.1, where the squared quantities are the dimensions of the manifolds and the quantities on the arrows are the corresponding codimensions. We point out especially the nontrivial conclusions concerning $O_{S R} \cap O_{S T}$ and $O_{R} \cap O_{S}$.

In fact, in a neighborhood of a $B K$-pair $\left(A_{c}, B_{c}\right)$, we have an adapted coordinate system $(S, \alpha, \gamma, \delta)$ of $\mathcal{M}^{*}$, where $S \in G l_{n}$ and $\alpha, \gamma, \delta$ are the parameters in Definition 2.3 , in a such way that:

$$
\begin{gathered}
O_{B K}=\{\delta=0\}, \quad O_{S T}=\{\delta=\alpha=0\} \\
O_{S R}=\{\delta=\gamma=0\}, \quad O_{S}=\{\delta=\alpha=\gamma=0\} .
\end{gathered}
$$

Clearly, $(\alpha, \beta)$ is a coordinate system of $O_{R}$, and one has proved that:

$$
O_{S} \cap O_{R}=\{\alpha=0\} .
$$

Moreover, if $\delta=0$, the parameters $\alpha, \gamma$ classify the $S T$-classes and the $S R$-classes, respectively, and the pair $(\alpha, \gamma)$, the $S$-classes. 


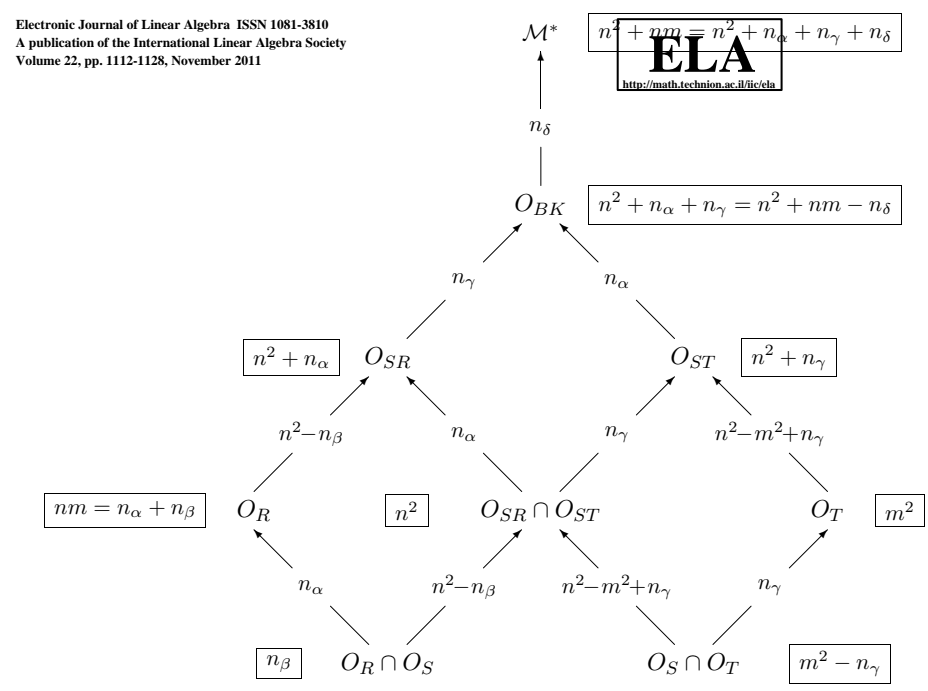

FIG. 5.1. Summary diagram.

5.2. Feedbacks. In control theory, feedbacks are used in different contexts, such as the study of conditioned invariant subspaces ([5]) or in the pole assignment problem, where any prescribed spectrum for $A+B R$ can be obtained provided that $(A, B)$ is controllable. It seems natural (see, for example, [16]) to study which other characteristics of $A$ can be changed by means of suitable feedbacks, and what kind of feedbacks are needed in each case. For instance, if one considers a $B K$-pair $\left(A_{c}, B_{c}\right)$, it is clear that any pole assignment can be solved using only block-diagonal feedbacks. Let us see that, from our study in the above sections, one has the following surprising conclusions:

- the $\beta$-feedbacks do not change any Jordan invariant, and any $\alpha$-feedback perturbs the Jordan type.

- any Jordan form near $A_{c}$ can be obtained by means of a suitable $\alpha$-feedback.

Indeed we have shown that the $\beta$-feedbacks do not change any Jordan invariant of $A_{c}$, because they are equivalent to changes of basis of the state space. On the other hand, we have proved that any $\alpha$-feedback perturbs the Jordan type of $A_{c}$. We notice that, from a "local" approach, these feedbacks form a miniversal deformation of $A_{c}$ in the sense of [2]. That is to say, that any Jordan form near $A_{c}$ can be obtained by means of a suitable $\alpha$-feedback or, even more, that they induce any local differentiable family of perturbations of $A_{c}$ in the space $\mathbb{C}^{n^{2}}$.

We have seen in Section 5.1 that these parameters also play an important role from the point of view of classifying pairs of matrices; the $\alpha$-feedbacks parameter- 
ize the $S T$-classes contained in $O_{B K}\left(A_{c}, B_{c}\right)$, and in particular they form an $S T$ miniversal deformation of $\left(A_{c}, B_{c}\right)$ in its $B K$-orbit. This role of $\alpha$-feedbacks as an $S T$-deformation, and their role as a Jordan-deformation in the above paragraph, is not evident. For instance, for $\alpha \neq \alpha^{\prime}$ (small, nonzero), the pairs $\left(A_{c}+A_{\alpha}, B_{c}\right)$ and $\left(A_{c}+A_{\alpha^{\prime}}, B_{c}\right)$ are not $S T$-equivalent, but the matrices $A_{c}+A_{\alpha}$ and $A_{c}+A_{\alpha^{\prime}}$ may be similar.

Finally, we remark the quite surprising fact that the number of $\beta$-parameters, $n_{\beta}$, (more precisely, the dimension of $O_{S} \cap O_{R}$ ) is just the codimension of $O_{S R}$ in $\mathcal{M}$. It is zero if and only if $k_{1}=k_{2}=\cdots=k_{m}$.

5.3. Dimension of orbits. Notice that

$$
\operatorname{dim} O_{B K}=n^{2}+m^{2}+n m-\operatorname{dim}\left(O_{S} \cap O_{T}\right)-\operatorname{dim}\left(O_{S} \cap O_{R}\right)
$$

(recall that $\operatorname{dim}\left(O_{T} \cap O_{R}\right)=0$ ). This gives a nice geometric interpretation:

- the dimension of $O_{B K}$ (as well as $O_{S T}$ and $O_{S R}$ ) depends only on the "overlaps" $O_{S} \cap O_{R}$ and $O_{S} \cap O_{T}$.

We have already seen that $\operatorname{dim} O_{S} \cap O_{R}=0$ if and only if $k_{1}=k_{2}=\cdots=k_{m}$. Then and only then, $O_{S} \cap O_{T}=O_{T}$ (or equivalently, $O_{S T}=O_{T}$ ) and $O_{S R}=O_{B K}$.

More generally, when $k_{1}-k_{m} \leq 1$, and only then, the $B K$-orbit has null codimension, or equivalently, it is open (hence, dense, from the Closed Orbit Lemma (see for example [10])).

When changing the controllability indices and the indices are not minorized, the dimension of the $B K$-orbits may be equal. Nevertheless, in these cases there is not a clear pattern between this dimension and the dimension of the $S T$-orbits or $S R$-orbits. See, for instance, Table 5.1.

\begin{tabular}{|c|c|c|c|}
\hline$k$ & $\operatorname{codim} O_{B K}$ & $\operatorname{codim} O_{S T}$ & $\operatorname{codim} O_{S R}$ \\
\hline $6,3,3,1$ & 10 & 47 & 15 \\
$5,5,2,1$ & 10 & 47 & 15 \\
$5,4,2,2$ & 6 & 47 & 11 \\
$4,4,4,1$ & 6 & 49 & 9 \\
$5,3,3,2$ & 4 & 47 & 9 \\
\hline \multicolumn{4}{|c|}{ TABLE 5.1 } \\
Dimension of Orbits
\end{tabular}




\section{REFERENCES}

[1] A.C. Antoulas. On canonical forms for linear constant systems. International Journal of Control, 33:95-122, 1981.

[2] V.I. Arnold. Matrices depending on parameters. Uspekhi Matematicheskikh Nauk, 26:101-114, 1971.

[3] J. Ferrer, M.I. García, and F. Puerta. Brunovsky local form of a holomorphic family of pairs of matrices. Linear Algebra and its Applications, 253:175-198, 1997.

[4] J. Ferrer, M.I. García, and F. Puerta. Regularity of the Brunovsky-Kronecker stratification. SIAM Journal on Matrix Analysis and Applications, 21:724-742, 2000.

[5] J. Ferrer, F. Puerta, and X. Puerta. Stratification and bundle structure of the set of conditioned invariant subspaces in the general case. Systems and Control Letters, 48:77-86, 2003.

[6] V. Guillemin and A. Pollack. Differential Topology. Prentice-Hall, Inc., Englewood Cliffs, NJ, USA, 1974.

[7] M. Hazewinkel. On the (internal) symmetry groups of linear dynamical systems. Groups, Systems, and Many Body Physics, (P. Kramer and M. Dal Cin, editors). Friedr. Vieweg \& Sohn, Braunschweig, 1980.

[8] U. Helmke. Topology if the moduli space for reachable linear dynamical systems: the complex case. Mathematical Systems Theory, 19:155-187, 1986.

[9] D. Hinrichsen and D. Prätzel-Wolters. A Jordan canonical form for reachable linear systems. Linear Algebra and its Applications, 122/123/124:489-524, 1989.

[10] J.E. Humphreys. Linear Algebraic Groups. Springer-Verlag, New York-Heidelberg, 1975.

[11] R.E. Kalman. Kronecker invariants and feedback. Ordinary Differential Equations. Academic Press, New York, 1972.

[12] J. Maroulas and S. Barnett. Canonical forms for time-invariant linear control systems: a survey with extensions. I. Single-input case. International Journal of Systems Science, 9:497-514, 1978.

[13] J. Maroulas and S. Barnett. Canonical forms for time-invariant linear control systems: a survey with extensions. II. Multivariable case. International Journal of Systems Science, 10:33-50, 1979.

[14] V.M. Popov. Invariant description of linear time-invariant controllable systems. SIAM Journal on Control and Optimization, 10:252-264, 1972.

[15] A. Tannenbaum. Invariance and System Theory: Algebraic and Geometric Aspects. SpringerVerlag, Berlin-New York, 1981.

[16] W.M. Wonham. Linear Multivariable Control: A Geometric Approach. Springer-Verlag, New York, 1979. 